CARDIOLOGY

\title{
Evaluation of Left Ventricular Performance: Is There a Gold Standard?
}

\author{
Konstantinos Dean Boudoulas ${ }^{a}$ Filippos Triposkiadis $^{b}$ Harisios Boudoulas ${ }^{c}$ \\ ${ }^{a}$ Department of Medicine, Division of Cardiovascular Medicine, The Ohio State University, Columbus, OH, USA; ${ }^{\mathrm{b}}$ Larissa \\ University Hospital, Larissa, Greece; 'Biomedical Research Foundation, Academy of Athens, Athens, Greece
}

Indices used to evaluate left ventricular (LV) performance in clinical practice (cardiac output, ejection fraction, other) are usually affected by many other factors such as preload, afterload, intrinsic or extrinsic inotropic effects, and LV geometry, just to mention a few $[1,2]$. In chronic aortic regurgitation (AR), preload, afterload and LV geometry are affected and for this reason, these factors should be taken into consideration when evaluating LV performance in these patients.

\section{Hemodynamics in Chronic AR}

$\mathrm{AR}$ is a unique condition; it is an isolated valve disease that results in both an increase in preload and afterload. The extra volume that regurgitates back into to the LV during diastole results in LV pressure overload and the ejected volume into the aorta during systole results in a large stroke volume and systolic hypertension. Pressure overload causes the thickening of the LV walls resulting in concentric hypertrophy; this is in addition to an already preceded eccentric hypertrophy. The LV dilatation (eccentric hypertrophy) and the con- centric hypertrophy, present in chronic AR, alter LV geometry, which in turn affects LV function $[3,4]$.

During the compensated phase of the disease, concentric hypertrophy (thick LV wall) results in a normalization of the LV wall stress. Stress $=\mathrm{P} \times \mathrm{r} / 2 \mathrm{~h}$, where $\mathrm{P}=$ pressure, $r=$ radius of the LV cavity and $h=L V$ wall thickness. During the compensated phase, the LV ejection fraction may be maintained despite a high afterload. As the disease progresses, however, in certain cases, the afterload may become as high as it is in aortic stenosis and the LV ejection fraction eventually will decrease. Certain patients with chronic AR may be asymptomatic, even when the LV ejection fraction decreases. Surgery in asymptomatic patients with AR and a decrease in LV ejection fraction is associated with a suboptimal outcome, as valve replacement at this stage of the disease may be too late $[3,4]$.

Several indices of LV function have been proposed including echocardiographic techniques, hemodynamic data, and resting and exercise ejection fraction, but due to the fact that in AR changes in preload, afterload and LV geometry often coexist, none of these indices in isolation appear to be perfect. For this reason,

\section{KARGER}

(C) 2018 S. Karger AG, Basel

E-Mail karger@karger.com

www.karger.com/crd
Harisios Boudoulas, MD

Biomedical Research Foundation Academy of Athens

E-Mail boudoulas@bioacademy.gr 
in certain cases, a combination of different indices has been used.

Dr. Borer et al. [5], in this issue of Cardiology, present the long-term outcomes in patients with severe chronic AR, where a combination of several parameters related to LV function were used prior to aortic valve replacement. After the exclusion criteria, 66 patients were studied. Patients were recruited from the Institute for Heart Valve Disease, which was established several decades ago in their Medical Center. LV ejection fraction at rest normalized for end-systolic stress and LV ejection fraction at peak exercise normalized for end-systolic stress at exercise were obtained in all patients [4-7]. From these measurements, changes in the LV ejection fraction from rest to exercise normalized for changes in end-systolic stress from rest to exercise were calculated. This index, defined by the authors as "contractility," was used to stratify patients as related to prognosis in 3 different groups. The values obtained from patients were compared to those obtained from 26 normal subjects [4]. During a 13 years follow-up period, 22 patients died. It was demonstrated that an abnormal response, as compared to normal, was associated with a poor prognosis [5]. The greater the deviation from normal values the worse the outcome. The authors concluded that determination of "contractility" in patients with chronic severe AR may be particularly useful in defining the optimal time for aortic valve replacement, since myocardial dysfunction may be present in these patients prior to symptom onset [5].

\section{Factors Affecting LV Performance with Emphasis on AR}

In addition to the intrinsic contractile state of myocardial cells, several other factors play a dominant role in modulating LV performance in an intact cardiovascular system not only in patients with various diseases including AR but in normal subjects as well. Among others, loading conditions, neurohumoral activation (especially sympathetic nervous system), and LV geometry play an important role $[1,2]$. The effects of these factors on LV function are briefly discussed.

\section{Preload}

In general, the relationship of end-diastolic volume to stroke volume and to LV ejection fraction is governed by the Frank-Starling mechanism, where LV performance

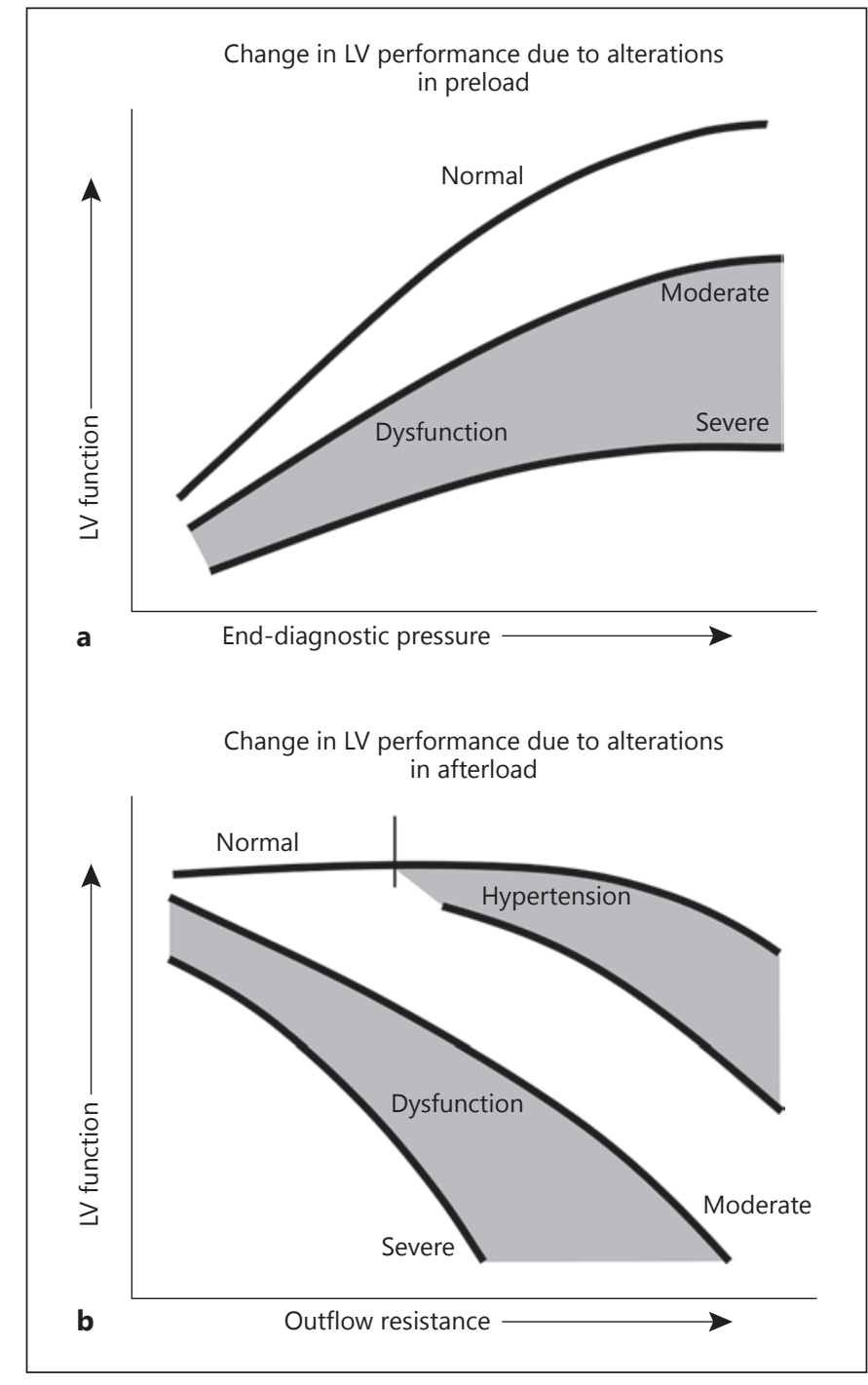

Fig. 1. a Effect of preload on left ventricular (LV) function. LV function curves in normal subjects and in patients with moderate and severe LV dysfunction. b Effect of afterload on LV function. LV function curves in normal subjects and in patients with moderate and severe LV dysfunction (modified from Binkley and Boudoulas [1]).

depends on the length of the muscle fiber at end-diastole (preload) $[1,8]$. In normal subjects, a small decrease in $\mathrm{LV}$ filling pressure results in a marked decrease in the LV ejection fraction and stroke volume. In contrast, in patients with significant LV dysfunction, a large decrease in an elevated end-diastolic pressure (preload) may result in little or no change in the stroke volume (Fig. 1a) [1]. It should be mentioned that for any given preload, the stroke volume or ejection fraction are also affected by myocardial contractility [1]. 


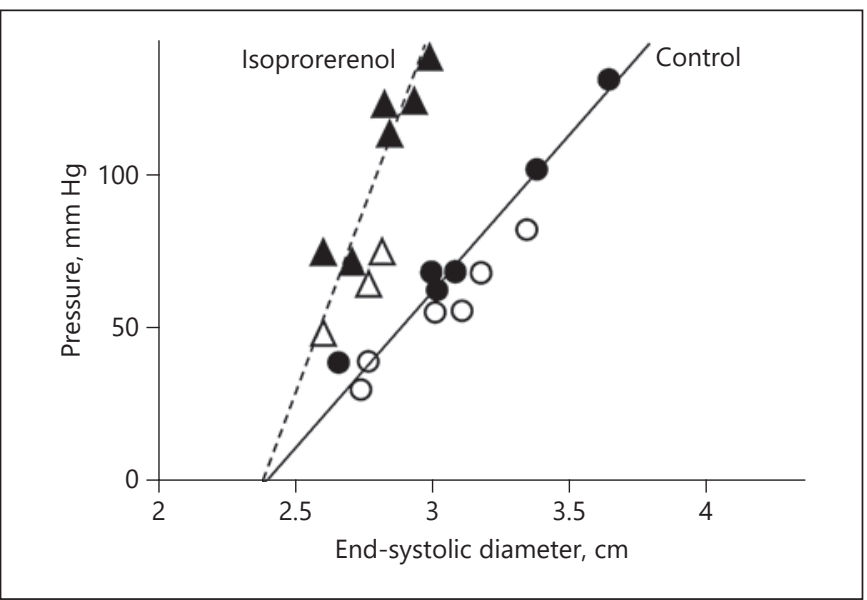

Fig. 2. Linear relationship of end-systolic pressure and end-systolic diameter. Note that with a positive inotropic intervention (isoproterenol infusion) at any given end-systolic pressure the endsystolic diameter is smaller indicating a positive inotropic effect (modified from [9]).

\section{Afterload}

The response to an increase in afterload is also different in the normal compared to failing LV. Despite increase resistance to LV ejection, stroke volume and ejection fraction remain unchanged in normal subjects and may slightly decrease in patients with arterial hypertension and normal LV function. In contrast, in patients with LV dysfunction, a significant decrease in LV ejection fraction and stroke volume occurs when afterload is increased (Fig. 1b) [1]. To correct for the effect that afterload may have on LV performance, the end-systolic pressure/endsystolic volume relationship has been proposed as another index of LV performance. There is a linear relationship between these 2 parameters (volume versus pressure) as can be seen by plotting LV end-systolic volumes or diameters with varying afterloads. As afterload increases, the end-systolic diameter increases as well (Fig. 2) [9]. Studies in the cardiac catheterization laboratory utilizing angiographic volumes plotted against varying end-systolic pressures have confirmed the linearity of this relationship in humans.

\section{Inotropy}

The effects of inotropic agents on LV performance have been well documented [1]. The effect of inotropic agents can also be seen in the end-systolic pressure-endsystolic volume relationship (Fig. 2) [9]. It is obvious that with isoproterenol infusion, the ventricle contracts to a smaller volume (diameter) for any given afterload.

\section{Geometry}

"The whole is greater than the sum of its parts" - Aristotle

LV ejection fraction has been used extensively in clinical practice to stratify patients with different diseases in high or low risk groups, classify patients with heart failure, and to assess the effect of therapeutic interventions [2]. LV ejection fraction, however, depends not only on the contractility of the cardiomyocytes, but also on the geometry of the LV chamber. It is well known for years that the cardiomyocytes have a limited range of shortening during LV systole. When measured in isolation, mammalian cardiomyocyte systolic shortening and thickening are approximately 15 and $8 \%$ respectively. With this degree of myocardial shortening and thickening, an ejection fraction of $60 \%$ or greater cannot be achieved without an appropriate orientation of cardiomyocytes in the wall of the LV cavity. From multiple studies, it has been shown that the most effective orientation of cardiomyocytes to provide an optimal ejection fraction is the helical or spiral formation [2].

In patients with chronic AR, especially when LV dilatation is present, the geometry of the LV chamber is changed substantially. Though the end-systolic volume corrected for end-systolic stress may partially correct for geometric changes, the precise effects of LV geometry on ventricular performance in patients with $A R$ remain to be defined.

\section{Measurement of LV Performance}

\section{Commonly Used Indices to Evaluate LV Performance} in Clinical Practice

Indices used to assess LV performance are based on the capacity of the heart to pump blood, its ability to generate force, the degree of chamber shortening with each contraction, and the temporal relationship of contraction. Based on these concepts, over the last several decades, cardiac output, LV pressures including LV dP/dt, LV ejection fraction, and systolic time intervals have been used. Today, however, in daily clinical practice, cardiac output, LV pressures, and LV ejection fraction are most commonly used. Because all these measurements are sensitive to loading conditions and LV geometry, in clinical research and in certain cases in clinical practice, in addition to the indices mentioned above, different combinations of these parameters such as LV pressure or stress to LV volume relationships have been used [1]. 


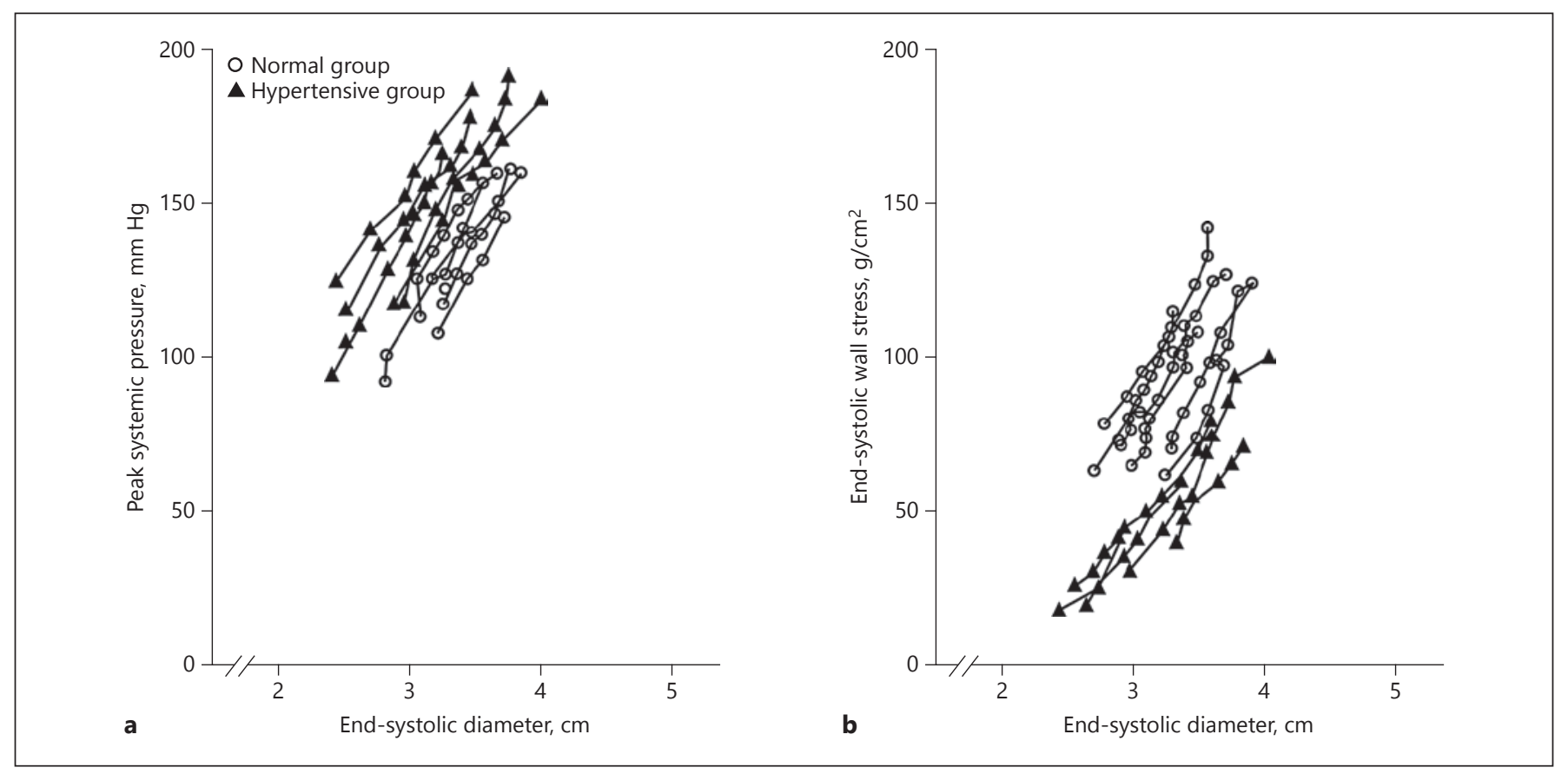

Fig. 3. Peak systolic pressure (a) and end-systolic stress (b) plotted against end-systolic diameters in normal subjects and patients with arterial hypertension and left ventricular (LV) hypertrophy. Mea-

\section{Pressure or Stress: LV Volume Relationship}

As it is mentioned above, the slope of the line relating end-systolic pressure to LV end-systolic volume has been used to assess LV performance. This relation does not only correct for changes occurring in afterload, but also it appears to be independent to preload. In LV hypertrophy, however, the relationship of end-systolic pressure to end-systolic volume alone may not accurately reflect LV function. This occurs because in LV hypertrophy, the thick LV walls result in a normalization of LV wall stress that compensates for the increase pressure. Thus, despite the high pressure, the end-systolic pressure to end-systolic volume ratios will be similar to those of normal subjects (Fig. 3a) [10]. In such a case, the relationship of end systolic stress to end-systolic volume (dimension) provides a more accurate index of LV performance because it corrects for LV thickness (Fig. 3b) [10]. The LV wall stress in relation to $L V$ ejection fraction also has been used to assess LV performance in patients with concentric hypertrophy (e.g., aortic stenosis). While in many patients the amount of hypertrophy that develops is adequate to normalize LV wall stress, in other cases, with a failure of adequate hypertrophy, the LV wall stress will increase and the ejection fraction will decline [11]. In the settings of regional wall motion abnormalities, echocardiogra- surement of stress/diameter relationship suggests decrease in inotropy in patients with arterial hypertension that is not indicated by the pressure/diameter relationship alone (modified from [10]).

phy or angiography may be not accurately estimate LV volumes. In the failing $\mathrm{LV}$, however, where alterations in afterload have a profound influence on LV performance, while changes in preload have minimal effect (Fig. 1), this relationship may represent an ideal measure of LV performance, but studies in this group of patients are limited [2].

\section{Clinical Pitfalls}

"Nothing is entirely true, but there is some truth in each aspect... We do fairly well with half-truths so long as we remember that there are half-truths." Dialogues of Alfred North Whitehead

(1953, as recorded by Lucian Price)

The evaluation of LV performance has been a research subject for more than a century. For years, studies were performed mostly in the research laboratories where many factors that influence LV function were individually controlled. Extrapolation of these laboratory techniques has comprised the basis for indices employed in clinical setting to assess LV function. Clinical scientists, however, are limited in that they are not afforded total control of the com- 
plex interactions that may alter LV performance. Consequently, one is forced to rely on indices that are not pure in their measurement of LV function. Much like the ancient alchemist who futilely attempted to arrive at the formula for gold, clinicians have for many years attempted to find the ideal index to assess LV performance that is independent of extrinsic factors. Although techniques for the evaluation of LV performance in clinical practice have become increasingly refined, there probably will never be an ideal index for this purpose. Each method has its limitations and advantages. In the clinical setting, we can only be aware of shortcomings of each technique and select the methods that are least sensitive to the factors that are most altered in a given setting [1].
Dr. Borer et al. [5] should be commended for this important work, where the best approach in patients with chronic AR has been used. This important work will help clinicians to define the optimal time for valve surgery in patients with chronic AR. Importantly, the study also provides insights into pathophysiologic mechanisms related to LV function and dysfunction in patients with chronic AR.

\section{Disclosure Statement}

The authors declare that they have no conflicts of interests to disclose.

\section{References}

1 Binkley PF, Boudoulas H: Measurement of myocardial inotropy. In Crdiotonic Drugs. A Clinical Survey. Leier CV ed., Marcel Dekker, Inc. New York, Basel 1986, pp 5-48.

2 Triposkiadis F, Giamouzis G, Boudoulas KD, et al: Left ventricular geometry as a major determinant of left ventricular ejection fraction: physiologic considerations and clinical implications. Eur J Heart Fail 2018;20:436444.

3 Bonow RD: Radionuclide angiography in the management of asymptomatic aortic regurgitation. Circulation 1991;84:1296-12302.

4 Borer JS, Hochreiter C, Harrold EM, et al: Prediction of indications for valve replacement among asymptomatic or minimally symptomatic patients with chronic aortic re- gurgitation and normal left ventricular performance. Circulation 1998;97:525-534.

5 Borer JS, Supino PG, Herrold E McM, et al: Survival after aortic valve replacement of aortic regurgitation: prediction from preoperative contractility measurement. Cardiology 2018, DOI 10.1159/000490848.

6 Reichek N, Wilson J, St John Sutton M, et al: Noninvasive determination of left ventricular end-systolic stress: validation of the method andinitial application. Circulation 1982;65: 99-108.

7 Gaasch WH, Carrol JD, Levine HJ, Criscitiello MG: Chronic aortic regurgitation: prognostic value of end-systolic diameter and enddiastolic diameter radius/thickness ratio, J Am Coll Cardiol 1983;1:775-782.
8 Patterson SW, Starling EH: On the mechanical factors which determine the output of the ventricles. J Physiol 1914;48:165-228.

9 Sagawa K, Suga H, Shoukas AA, Bakalar KM: End-systolic pressure/volume ratio: a new index of ventricular contractility. AM J Cardiol 1977;40:746-753.

10 Takahashi M, Sasayama C, Kawai C, Kotoura N: Contractile performance of the hypertrophied ventricle in patients with systemic hypertension. Circulation 1980;62:116126.

11 Carabello AA, Green LH, Grossman W, et al: Hemodynamic determinants of prognosis of aortic valve replacement in critical aortic stenosis and advanced congestive heart failure. Circulation 1980;62:42-48. 